\title{
A patient perspective of the impact of medication side effects on adherence: results of a cross- sectional nationwide survey of patients with schizophrenia
}

\author{
Marco DiBonaventura ${ }^{1 *}$, Susan Gabriel${ }^{2}$, Leon Dupclay ${ }^{2}$, Shaloo Gupta ${ }^{1}$ and Edward Kim²
}

\begin{abstract}
Background: Antipsychotic medications often have a variety of side effects, however, it is not well understood how the presence of specific side effects correlate with adherence in a real-world setting. The aim of the current study was to examine the relationship between these variables among community-dwelling patients with schizophrenia.

Methods: Data were analyzed from a 2007-2008 nationwide survey of adults who self-reported a diagnosis of schizophrenia and were currently using an antipsychotic medication $(N=876)$. The presence of side effects was defined as those in which the patient reported they were at least "somewhat bothered". Adherence was defined as a score of zero on the Morisky Medication Adherence Scale. To assess the relationship between side effects and adherence, individual logistic regression models were fitted for each side effect controlling for patient characteristics. A single logistic regression model assessed the relationship between side effect clusters and adherence. The relationships between adherence and health resource use were also examined.
\end{abstract}

Results: A majority of patients reported experiencing at least one side effect due to their medication (86.19\%). Only $42.5 \%$ reported complete adherence. Most side effects were associated with a significantly reduced likelihood of adherence. When grouped as side effect clusters in a single model, extra pyramidal symptoms (EPS)/agitation (odds ratio $(O R)=0.57, p=0.0007)$, sedation/cognition $(O R=0.70, p=0.033)$, prolactin/endocrine $(O R=0.69, p=$ $0.0342)$, and metabolic side effects $(O R=0.64, p=0.0079)$ were all significantly related with lower rates of adherence. Those who reported complete adherence to their medication were significantly less likely to report a hospitalization for a mental health reason $(\mathrm{OR}=0.51, \mathrm{p}=0.0006)$, a hospitalization for a non-mental health reason $(\mathrm{OR}=0.43, \mathrm{p}=0.0002)$, and an emergency room (ER) visit for a mental health reason ( $O R=0.60, p=0.008)$.

Conclusions: Among patients with schizophrenia, medication side effects are highly prevalent and significantly associated with medication nonadherence. Nonadherence is significantly associated with increased healthcare resource use. Prevention, identification, and effective management of medication-induced side effects are important to maximize adherence and reduce health resource use in schizophrenia.

\section{Background}

Schizophrenia is a chronic, often debilitating psychiatric illness with a lifetime prevalence of approximately $1 \%$ of the US population [1,2]. Typically manifesting in late adolescence or early adulthood, schizophrenia can

\footnotetext{
* Correspondence: marco.dibonaventura@kantarhealth.com 'Health Sciences Practice, Kantar Health, 11 Madison Avenue, 12th Floor, New York, NY, USA

Full list of author information is available at the end of the article
}

disturb perception, cognition, emotions, and behavior [3]. In addition to pronounced effects on the well-being of patients [4] and their families [5], schizophrenia also exacts an economic burden, estimated at almost $\$ 63$ billion per year in the US in 2002 [6].

Numerous antipsychotic medications are available with demonstrated efficacy in reducing the acute symptoms of schizophrenia, improving the well-being of patients, and enabling some to live more productive

\section{Ciomed Central}


lives [7]. However, adherence and persistence to these medications are important to receive optimal benefits. A review of dropout rates in clinical trials found that $28 \%$ $55 \%$ of schizophrenia patients drop out of clinical trials before the study is complete; dropout rates were higher with classic antipsychotic medications compared with second generation antipsychotic medications due to side effects [8]. In the Clinical Antipsychotic Trial of Interventional Effectiveness (CATIE) study, $74 \%$ of patients discontinued their initial study medication before 18 months [9]. Approximately half of patients with schizophrenia take $70 \%$ or less of their medication [10]. Inadequate adherence to antipsychotic medications increases the risk of relapse and associated healthcare utilization and costs [11-13]. A review by Sun et al. (2007) estimated that antipsychotic nonadherence in the US was responsible for between $\$ 1.4$ and $\$ 1.8$ billion in rehospitalization costs alone [11].

Studies have suggested that antipsychotic medication side effects are associated with lower levels of adherence $[14,15]$. Clinicians' ratings of side effects are also associated with treatment discontinuation [16]. Specifically, side effects such as medication-related obesity, distress over weight gain, and cognitive impairment have been associated with increased rates of nonadherence $[14,15,17,18]$. Although other studies have examined adherence in real-world settings [18], few have studied the relationship between specific side effects and nonadherence. Moreover, there are no studies assessing the relationship between patient-reported side effects and self-reported adherence. This patient perspective is valuable as it provides insight into how the perception of side effects is associated with specific non-adherent behaviors; something that cannot be obtained from objective assessments of adherence. The primary aim of the current study is to assess the relationship between patient-reported antipsychotic side effects and selfreported medication adherence in a community-dwelling sample of patients with schizophrenia. A secondary aim is to assess the relationship between medication adherence and self-reported health resource utilization.

\section{Methods}

\section{Data source}

We analyzed an existing cross-sectional dataset of patients with schizophrenia $(\mathrm{N}=1,083)$. This original survey, conducted between December 2007 and February 2008, was initiated to understand the treatments, attitudes, health behaviors, and health outcomes among patients with schizophrenia. The data generated from this survey has been used in several previous studies, each of which has outlined the methods in some detail $[4,19]$. Briefly, patients were convenience sampled in one of two ways to participate in a self-administered survey to create the dataset: (1) patients who reported having schizophrenia in an Internet-based consumer panel (Lightspeed Research Ailment Panel) were randomly sent an invitation to participate in a web-based questionnaire via email, and (2) patients were also recruited from grassroots campaigns and newspaper advertising to arrive at a central interview facility to take a paper copy of the survey instrument.

The Lightspeed Research Ailment Panel is an opt-in Internet panel which recruits its members through a variety of online sources (e.g., online support groups, etc.). Patients with schizophrenia who join the panel provide detailed demographic information and agree to participate in a modest number of periodic Internetbased surveys. No sampling frame was used for patients who were recruited outside the Internet panel. Patients who responded to the study advertisements were phone screened for eligibility. Those who reported they were diagnosed with schizophrenia by a healthcare professional, were 18 years of age or older, and were able to read and write English were eligible for this study.

For patients taking the survey online $(\mathrm{N}=157)$, an email address was provided for patients to ask any questions they may have about the survey. Similarly, for patients taking the survey at an interview site $(\mathrm{N}=926)$, a facilitator was present to answer any questions. All patients, regardless of methodology, provided informed consent and were compensated for their participation. Patients who completed the survey online were compensated in the form of points, which can be exchanged for small prizes through Lightspeed Research. Patients who completed the survey offline received a $\$ 50$ check as compensation. The study protocol and questionnaire were reviewed and approved by Essex IRB (Lebanon, $\mathrm{NJ})$.

\section{Sample}

The study sample was limited to patients who reported that they were currently taking a prescription medication for schizophrenia $(\mathrm{N}=876)$.

\section{Study measures}

\section{Patient characteristics}

Patient characteristics consisted of demographic data and healthcare characteristics. All demographic data, except age, were assessed as categorical variables and included: gender (female vs. male), ethnicity (non-white vs. white) marital status (married/committed relationship vs. all else), education (some college education or higher vs. no college education/less than college education), employment status (employed full-time or parttime vs. not employed), insurance status (insured vs. uninsured), and poverty (annual household income less than $\$ 20,000$ vs. income $\$ 20,000$ or greater). Health 
characteristics consisted of the total number of comorbid medical conditions, which included type I and type II diabetes, high blood pressure, high cholesterol, heart disease, migraine, liver disease, and HIV.

\section{Medication side effects}

Medication side effects were self reported. Patients currently taking a prescription medication to treat their schizophrenia were asked, "In the past month, have you experienced any of the following side effects from your medication?". Among patients who responded "yes" to any of the listed side effects, they were asked "how bothered are you by these side effects?". Responses included "not at all bothered", "not very bothered", "somewhat bothered", "very bothered", and "extremely bothered". Our pre-specified definition of a side effect included any side effect in which the patient was somewhat, very, or extremely bothered. Side effects were also clustered into five pre-specified categories: "extra pyramidal symptoms (EPS)/agitation" (insomnia, restlessness/feeling jittery, agitation, and tremors); "sedation/cognition" (sedation, difficulty thinking or concentrating, sleepiness, and dizziness); "prolactin/endocrine" (decreased interest in sex, sexual dysfunction, difficult or painful menstrual periods, male breast enlargement or secretions); "metabolic" (weight gain, increase in blood glucose level); and "gastrointestinal (GI)" (nausea/vomiting and constipation). Post-hoc sensitivity analyses used a higher threshold for side effect definition of very or extremely bothered and removed "agitation" from the EPS/agitation cluster due to the potential confounding of agitation and restlessness associated with akathisia.

\section{Medication adherence}

Adherence to medications was assessed using the fouritem Morisky Medication Adherence Scale (MMAS) [20], an instrument which has previously shown to have strong evidence for reliability and validity [20]. The MMAS items include the presence or absence of the following non-adherent behaviors: forgetting to take medication, careless at times about taking medication, stopping medication when feeling better, and stopping medication when feeling worse. For this study we used a threshold of one or more items to identify nonadherence; adherence was classified as reporting "no" for all items. This approach has been reported in previous schizophrenia research [19].

\section{Health resource use}

Health resource use included self-reported emergency room (ER) visits (for mental and non-mental healthrelated reasons) and hospitalizations (for mental and non-mental health-related reasons) in the prior six months. Each of these four outcomes was defined dichotomously as yes ( 1 or more visits) vs. no (0 visits). Prior research has suggested patient-reported measures of resource use show good evidence for validity in comparison with objective measures [21-25].

\section{Statistical analysis}

Descriptive statistics were calculated to describe the patient characteristics, side effect, and medication adherence for the entire sample. Unadjusted comparisons of patient characteristics between adherent and nonadherent groups were conducted using chi-square tests and ANOVA tests for categorical and continuous variables, respectively.

To examine the relationship between side effects and nonadherence, a logistic regression model was fitted for each side effect adjusting for age, gender, ethnicity, education, household income, and number of comorbidities. A single logistic regression model was also fitted using the five pre-specified side effect clusters, controlling the same covariates. To assess the relationship between adherence and health resource use, a logistic regression model was fitted for each of the resource use outcomes, controlling for age, gender, marital status, ethnicity, education, household income, insurance and the number of comorbidities. For all regression models, an a priori threshold for statistical significance was set at $\mathrm{p}<0.05$.

The individual side effect models were also fitted using the more restrictive definition of side effect presence ("very bothered" or "extremely bothered"). The symptom cluster model was also fitted after removing "agitation" from the EPS/agitation cluster in order to assess the robustness of that cluster.

\section{Results}

The study sample consisted of 876 patients with schizophrenia who reported they were currently taking a medication to treat their condition. The mean age of the sample was 43 years, approximately half of the sample was male, and $61 \%$ was white (see Table 1). Over half of the sample was unemployed and $71.7 \%$ were taking atypical antipsychotics. The most commonly reported side effects that were at least moderately bothersome included difficulty thinking/concentrating (32.2\%), restlessness/feeling jittery (28.2\%), insomnia (28.4\%), weight gain $(25.8 \%)$, and sleepiness $(25.1 \%)$ (see Table 2 ).

The frequency of nonadherent behaviors (individual items of the MMAS) are presented in Figure 1. Nearly half of patients reported that they sometimes forget to take their medication (48.4\%). Only $42.5 \%$ of patients responded "no" to all four nonadherent behaviors in the MMAS. Table 3 summarizes bivariate comparisons between characteristics of adherent and nonadherent patients. Fewer adherent patients were married or had any college education. There were no other significant differences in patient characteristics between groups. 
Table 1 Sample demographics $(\mathbf{N}=\mathbf{8 7 6})$

\begin{tabular}{|c|c|c|}
\hline & $n$ & $\%$ \\
\hline \multicolumn{3}{|l|}{ Gender } \\
\hline Male & 432 & 49.3 \\
\hline Female & 444 & 50.7 \\
\hline \multicolumn{3}{|l|}{ Ethnicity } \\
\hline White & 536 & 61.2 \\
\hline Non-white & 340 & 38.8 \\
\hline \multicolumn{3}{|l|}{ Education } \\
\hline Less than college & 421 & 48.1 \\
\hline Some college or higher & 455 & 51.9 \\
\hline \multicolumn{3}{|l|}{ Household income } \\
\hline Less than $\$ 20,000$ & 506 & 57.8 \\
\hline$\$ 20,000$ or more & 370 & 42.2 \\
\hline \multicolumn{3}{|l|}{ Marital status } \\
\hline Single & 665 & 75.9 \\
\hline Married/living with partner & 211 & 24.1 \\
\hline \multicolumn{3}{|l|}{ Insurance } \\
\hline Managed care (HMO, PPO) & 241 & 27.5 \\
\hline Medicare & 364 & 41.6 \\
\hline Veterans Affairs Medical Center & 41 & 4.7 \\
\hline State Medicaid (MediCal for CA residents) & 350 & 40.0 \\
\hline No health insurance & 61 & 7.0 \\
\hline Don't know & 16 & 1.8 \\
\hline \multicolumn{3}{|l|}{ Employment } \\
\hline Employed full time & 129 & 14.7 \\
\hline Employed part time & 163 & 18.6 \\
\hline Student & 29 & 3.3 \\
\hline Retired & 70 & 8.0 \\
\hline Not employed & 484 & 55.3 \\
\hline \multicolumn{3}{|l|}{ Atypical Medication } \\
\hline \multirow[t]{2}{*}{ On a atypical medication } & 628 & 71.7 \\
\hline & Mean & SD \\
\hline Age & 43.03 & 11.80 \\
\hline Number of comorbidities & 1.27 & 1.30 \\
\hline
\end{tabular}

The results of the logistic regression models for each side effect are presented in Figure 2. Most of the side effects assessed were significantly associated with a decreased likelihood of medication adherence. Sensitivity analyses using the more restrictive definition of side effect presence did not change overall model results with respect to significance or magnitude (see Additional file 1: Figure S1).

Table 4 summarizes results of the logistic regression model using five side effect clusters. Younger age, unemployment, and higher education were all associated with a lower likelihood of adherence. Similarly, all of the side effect clusters except GI were associated with a lower likelihood of adherence. The EPS/agitation cluster had the strongest effect, with a $43 \%$ reduction in odds of being adherent, followed by metabolic side effects with a $36 \%$ reduction. Results of the sensitivity analysis
Table 2 Medication side effects reported by current medication users $(\mathbf{N}=\mathbf{8 7 6})$

\begin{tabular}{lcc}
\hline & \multicolumn{2}{c}{$\begin{array}{c}\text { Side effect present and } \\
\text { bothersome }\end{array}$} \\
\hline Difficulty thinking/concentrating & $\mathbf{n}$ & $\%$ \\
Restlessness/feeling jittery & 349 & 32.2 \\
Insomnia & 305 & 28.2 \\
Sleepiness & 307 & 28.4 \\
Weight gain & 272 & 25.1 \\
Decreased interest in sex & 279 & 25.8 \\
Agitation & 223 & 20.6 \\
Sedation & 240 & 22.2 \\
Dizziness & 173 & 16.0 \\
Constipation & 193 & 17.8 \\
Tremors & 175 & 16.2 \\
Sexual dysfunction & 142 & 13.1 \\
Nausea/vomiting & 136 & 12.6 \\
Difficult/painful menstrual periods & 110 & 10.2 \\
Male breast enlargement or secretions & 51 & 4.7 \\
Increased in blood glucose level & 9 & 1.4 \\
\hline
\end{tabular}

removing agitation from the EPS/agitation cluster did not affect the significance or magnitude of the effect (see Additional file 2: Table S1).

Patients reporting complete medication adherence were significantly less likely to report a hospitalization for a mental health reason $(\mathrm{OR}=0.51$; $95 \% \mathrm{CI}$ : 0.35 $0.75, \mathrm{p}=.0006)$, a hospitalization for a non-mental health reason $(\mathrm{OR}=0.43$; 95\%CI: 0.28-0.67, $\mathrm{p}=.0002)$, or an ER visit for a mental health reason $(\mathrm{OR}=0.60$; 95\%CI: 0.41-0.87, $\mathrm{p}=.008$ ).

\section{Discussion}

In this cross-sectional survey of patients in the US with schizophrenia, nearly $80 \%$ of patients reported at least one side effect that was at least somewhat bothersome

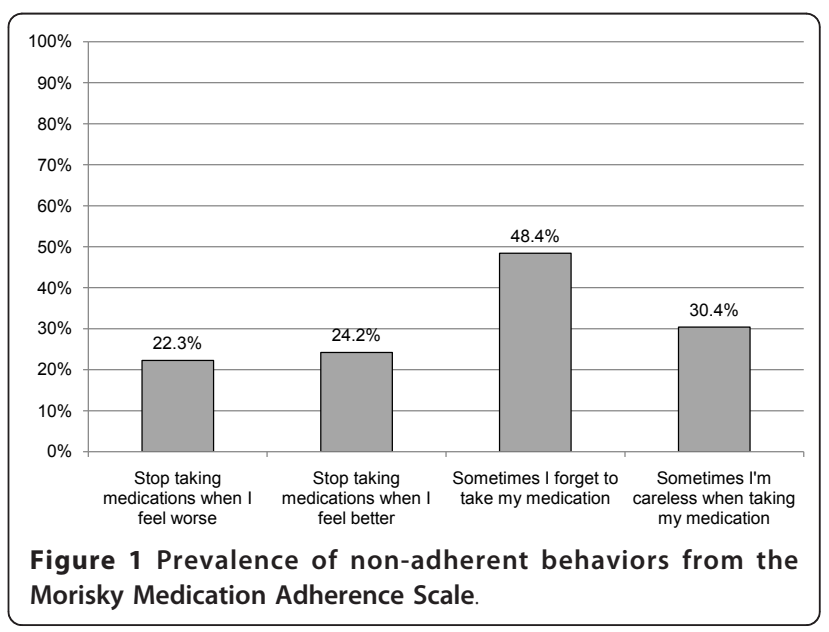


Table 3 Sociodemographic and patient characteristic differences between those adherent and non-adherent with their medication for their schizophrenia

\begin{tabular}{|c|c|c|c|c|c|}
\hline & \multicolumn{2}{|c|}{ Non-adherent $(n=504)$} & \multicolumn{2}{|c|}{ Adherent $(n=372)$} & \multirow[b]{2}{*}{$\mathrm{p}$} \\
\hline & $\mathrm{n}$ & $\%$ & $\mathrm{n}$ & $\%$ & \\
\hline Female & 260 & 51.6 & 184 & 49.5 & 0.5344 \\
\hline Married & 138 & 27.4 & 73 & 19.6 & 0.0069 \\
\hline Non-white & 192 & 38.1 & 148 & 39.8 & 0.6127 \\
\hline Some college or higher & 283 & 56.2 & 172 & 46.2 & 0.0037 \\
\hline Household income $\$ 20,000$ or more & 226 & 44.8 & 144 & 38.7 & 0.0685 \\
\hline Insured & 455 & 90.3 & 344 & 92.5 & 0.2487 \\
\hline \multirow[t]{2}{*}{ Employed } & 156 & 31.0 & 136 & 36.6 & 0.0838 \\
\hline & Mean & SD & Mean & SD & $p$ \\
\hline Age & 42.40 & 12.02 & 43.89 & 11.46 & 0.0651 \\
\hline Number of comorbidities & 1.34 & 1.29 & 1.18 & 1.31 & 0.0861 \\
\hline
\end{tabular}

to them, and less than half of patients reported complete adherence to their medications. There were few differences in the characteristics of adherent and nonadherent patients. However, we observed consistently strong relationships between medication side effects and nonadherence. Our finding that cognitive-related side effects and weight gain were associated with nonadherence is consistent with prior research $[17,18]$.

EPS/agitation-related side effects were the most strongly associated with nonadherence, and were commonly reported. This is a striking finding because atypical antipsychotics are generally thought to have lower risk for EPS compared to typical antipsychotics [26,27].

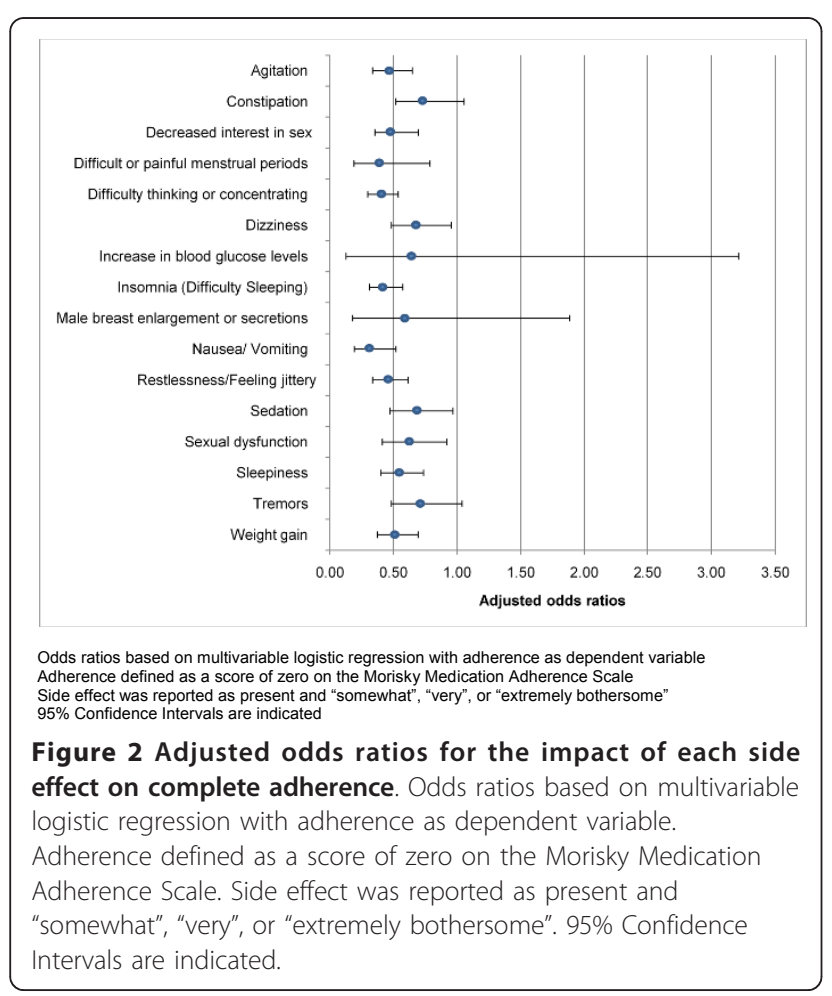

Though, it should be noted, not all patients in this study were on an atypical medication the vast majority were. Both EPS and endocrine side effects of antipsychotic medications are mediated by dopamine receptor 2 (D2) blockade [28]. The mechanism behind metabolic side effects to antipsychotics is less clear, but may be related to histaminergic blockade, which is also implicated in sedative side effects $[28,29]$.

Aside from side effects, a few other variables were significantly associated with adherence. Specifically, patients who were older, less educated, and employed were more likely to be adherent. Prior evidence has suggested that longer illness duration is associated with greater adherence [30]; therefore, age may be serving as a proxy for years diagnosed. Employment may be serving as a proxy for level of functioning (i.e., patients with improved functioning are more likely to be in the labor force), as poor disease insight has been shown to

Table 4 The relationship between side effect clusters and complete medication adherence

\begin{tabular}{lcccc}
\hline & OR & $\begin{array}{c}\mathbf{9 5 \%} \\
\text { LCL }\end{array}$ & $\begin{array}{c}\mathbf{9 5 \%} \\
\text { UCL }\end{array}$ & $\mathbf{p}$ \\
\hline Female & 1.08 & 0.80 & 1.45 & 0.6209 \\
Married & 0.73 & 0.51 & 1.06 & 0.0942 \\
Age & 1.02 & 1.00 & 1.03 & 0.0283 \\
Non-White & 0.99 & 0.73 & 1.33 & 0.9244 \\
Some college or higher & 0.72 & 0.53 & 0.97 & 0.0324 \\
Insured & 1.33 & 0.79 & 2.24 & 0.2821 \\
Employed & 1.45 & 1.03 & 2.03 & 0.0313 \\
Household income \$20,000 or & 0.83 & 0.60 & 1.15 & 0.264 \\
more & & & & \\
Number of comorbidities & 0.96 & 0.86 & 1.09 & 0.549 \\
Agitation/EPS & 0.57 & 0.41 & 0.78 & 0.0007 \\
Sedation/Cognition & 0.70 & 0.50 & 0.97 & 0.0331 \\
Prolactin/Endrocrine & 0.69 & 0.49 & 0.97 & 0.0342 \\
Metabolic & 0.64 & 0.46 & 0.89 & 0.0079 \\
Gl & 0.79 & 0.55 & 1.11 & 0.1729 \\
\hline
\end{tabular}


be associated with poorer adherence [30]. Interestingly, few studies have uncovered a relationship between education and adherence. Because of the lack of support in the literature, it is possible education also serves as a proxy for another unmeasured variable (e.g., negative attitudes toward medications), though additional research is warranted.

Consistent with previous studies [11], nonadherence is a significant risk factor for hospital and emergency room use. In our study, we found that both mental health and non-mental health hospital use was increased in nonadherent patients. Antipsychotic medications demonstrate high variability in their risk of inducing various side effects, and this may be mediated by differential affinities for D2, 5HT2A, and other receptors $[28,29]$. Clinicians may be faced with the challenge of choosing between medications with a lower risk for EPS yet higher risk for metabolic complications, and vice versa, but we find that both side effects are associated with significant nonadherence that may lead to both psychiatric and non-psychiatric hospitalizations. Therefore, preventing, identifying, and minimizing the frequency and severity of medicationrelated side effects may lead to greater adherence and fewer hospitalizations.

\section{Limitations}

This study has several limitations. Because all data were self-reported, diagnoses, treatments, adherence levels, and healthcare resource utilization were not confirmed by clinicians, patient records, or administrative claims data. Patients may have either underreported or overreported their experience with side effects (perhaps attributing a medication side effect to a symptom of a comorbid condition or vice versa) and their level of adherence. However, this patient perspective can still be valuable. Regardless of the true reason for the experienced "side effect", a patient's perception of the reason for the side effect may be more important in predicting adherence. Even if the side effect is not due to their schizophrenia medication, attributing it as such can result in greater non-adherence.

The cross-sectional design prevents robust ascertainment of causality. Although the hypothesis was that the presence of side effects leads to greater non-adherence it is possible that non-adherence (particularly sudden medication suspension followed by a full dose) can increase the presence of side effects. It is also possible that increasing adherence may lead to more side effects.

It is possible that unobserved confounding may have influenced the observed results. For example, severity of schizophrenia, polypharmacy, complexity of medication regimen, medication costs, among other variables, are likely associated with non-adherence but were not included in the current study. Similarly, limited information was available with respect to the number of nonantipsychotic medications. The greater the number of medications, the more difficult it may be for patients to determine which side effects are due to which treatments. Finally, the use of a convenience sample may have resulted in a sample that does not generalize to the community-dwelling population of patients with schizophrenia, as all patients were willing and functionally able to participate in survey research. The usage of both online and offline sample sources was implemented to ensure variability in patient types but these patient types may not be representative of the larger population.

\section{Conclusions}

Side effects of antipsychotic medications are highly prevalent and significantly associated with lower adherence, which is associated with increased healthcare resource use. Prevention of, monitoring for, and minimizing medication side effects may lead to better adherence and improved outcomes.

\section{Additional material}

Additional file 1: Adjusted odds ratios for the impact of each side effect on complete adherence with more restrictive definition of side effect presence.

Additional file 2: The relationship between side effect clusters and complete medication adherence, removing agitation from the agitation/EPS cluster.

\section{Abbreviations}

CATIE: Clinical antipsychotic trial of interventional effectiveness; IRB: Institutional review board; EPS: Extra pyramidal symptoms; Gl: Gastrointestinal; MMAS: Morisky medication adherence scale; ER: Emergency room; ANOVA: Analysis of variance; D2: Dopamine receptor 2.

\section{Acknowledgements}

The authors would like to thank Dr. Jeffrey Vietri for his contribution in assisting with the literature review. Dr. Vietri is an employee of Kantar Health. The authors would also like to thank Dr. Safiya Abouzaid who provided comments on the various drafts of this paper.

\section{Author details}

${ }^{1}$ Health Sciences Practice, Kantar Health, 11 Madison Avenue, 12th Floor, New York, NY, USA. ${ }^{2}$ Health Economics and Outcomes Research, Novartis Pharmaceutical Corporation, East Hanover, NJ, USA.

\section{Authors' contributions}

All authors (MD, SGa, LD, SGu, and EK) helped contribute to the conception and design of the current study. MD and SGu performed the statistical analyses. All authors helped contribute to the interpretation of the results. MD and SGu drafted the manuscript. All authors provided critical revisions to the various manuscript drafts. All authors read and approved the final manuscript

\section{Competing interests}

The nationwide survey reported here was completed by Kantar Health. Novartis Pharmaceutical Corporation purchased access to the data of the survey and funded the analysis and preparation of this manuscript. Ms. 
Gupta and Dr. DiBonaventura are current employees of Kantar Health; Ms. Gabriel and Drs. Dupclay and Kim are current employees of Novartis Pharmaceutical Corporation.

Received: 22 September 2011 Accepted: 20 March 2012 Published: 20 March 2012

\section{References}

1. Kendler KS, Gallagher TJ, Abelson JM, Kessler RC: Lifetime prevalence, demographic risk factors, and diagnostic validity of nonaffective psychosis as assessed in a US community sample. The National Comorbidity Survey. Arch Gen Psychiatry 1996, 53:1022-1031.

2. Goldner EM, Hsu L, Waraich P, Somers JM: Prevalence and incidence studies of schizophrenic disorders: a systematic review of the literature. Can J Psychiatry 2002, 47:833-843.

3. Messias EL, Chen CY, Eaton WW: Epidemiology of schizophrenia: review of findings and myths. Psychiatr Clin North Am 2007, 30:323-338.

4. DiBonaventura MD, Panish J, Kenworthy D, Wagner J-S, Dirani R: The association of well-being, productivity and resource use among community-dwelling patients with schizophrenia using atypical antipsychotics. J Pharmaceut Health Serv Res 2010, 1:181-187.

5. Caqueo-Urizar A, Gutierrez-Maldonado J, Miranda-Castillo C: Quality of life in caregivers of patients with schizophrenia: a literature review. Health Qual Life Outcomes 2009, 7:84.

6. Wu EQ, Birnbaum HG, Shi L, Ball DE, Kessler RC, Moulis M, Aggarwal J: The economic burden of schizophrenia in the United States in 2002. J Clin Psychiatry 2005, 66:1122-1129.

7. Liberman RP, Kopelowicz A, Ventura J, Gutkind D: Operation criterial and factors related to recovery from schizophrenia. Int Rev Psychiatry 2002, 14:256-272.

8. Kemmler G, Hummer M, Widschwendter C, Fleischhacker WW: Dropout rates in placebo-controlled and active-control clinical trials of antipsychotic drugs: a meta-analysis. Arch Gen Psychiatry 2005, 62:1305-1312.

9. Lieberman JA, Stroup TS, McEvoy JP, Swartz MS, Rosenheck RA, Perkins DO, Keefe RS, Davis SM, Davis CE, Lebowitz BD, et al: Effectiveness of antipsychotic drugs in patients with chronic schizophrenia. N Engl J Med 2005, 353:1209-1223.

10. Goff DC, Hill M, Freudenreich O: Strategies for improving treatment adherence in schizophrenia and schizoaffective disorder. J Clin Psychiatry 2010, 71(Suppl 2):20-26.

11. Sun SX, Liu GG, Christensen DB, Fu AZ: Review and analysis of hospitalization costs associated with antipsychotic nonadherence in the treatment of schizophrenia in the United States. Curr Med Res Opin 2007, 23:2305-2312.

12. Nicholl D, Akhras KS, Diels J, Schadrack J: Burden of schizophrenia in recently diagnosed patients: healthcare utilisation and cost perspective. Curr Med Res Opin 2010, 26:943-955.

13. Knapp M, Locklear J, Jarbrink K: Impact of psychotic relapse definitions in assessing drug efficacy and costs: comparison of quetiapine $\mathrm{XR}$, olanzapine and paliperidone ER. Curr Med Res Opin 2009, 25:1593-1603.

14. Barbui C, Kikkert M, Mazzi MA, Becker T, Bindman J, Schene A, Nose M, Helm H, Thornicroft G, Tansella M: Comparison of patient and clinician perspectives in the assessment of antipsychotic medication adherence. Psychopathology 2009, 42:311-317.

15. Burton SC: Strategies for improving adherence to second-generation antipsychotics in patients with schizophrenia by increasing ease of use. J Psychiatr Pract 2005, 11:369-378.

16. Fenton WS, Blyler CR, Heinssen RK: Determinants of medication compliance in schizophrenia: empirical and clinical findings. Schizophr Bull 1997, 23:637-651.

17. Weiden PJ, Mackell JA, McDonnell DD: Obesity as a risk factor for antipsychotic noncompliance. Schizophr Res 2004, 66:51-57.

18. Ascher-Svanum H, Zhu B, Faries D, Lacro JP, Dolder CR: A prospective study of risk factors for nonadherence with antipsychotic medication in the treatment of schizophrenia. J Clin Psychiatry 2006, 67:1114-1123.

19. Kim E, Gupta S, Bolge S, Chen CC, Whitehead R, Bates JA: Adherence and outcomes associated with copayment burden in schizophrenia: a crosssectional survey. J Med Econ 2010, 13:185-192.

20. Morisky DE, Green LW, Levine DM: Concurrent and predictive validity of a self-reported measure of medication adherence. Med Care 1986, 24:67-74.
21. Goossens ME, Rutten-van Molken MP, Vlaeyen JW, van der Linden SM: The cost diary: a method to measure direct and indirect costs in costeffectiveness research. J Clin Epidemiol 2000, 53:688-695.

22. Petrou S, Murray L, Cooper P, Davidson LL: The accuracy of self-reported healthcare resource utilization in health economic studies. Int J Technol Assess Health Care 2002, 18:705-710.

23. Pinto D, Robertson MC, Hansen P, Abbott JH: Good agreement between questionnaire and administrative databases for health care use and costs in patients with osteoarthritis. BMC Med Res Methodol 2011, 11:45.

24. Raina P, Torrance-Rynard V, Wong M, Woodward C: Agreement between self-reported and routinely collected health-care utilization data among seniors. Health Serv Res 2002, 37:751-774.

25. Ritter PL, Stewart AL, Kaymaz H, Sobel DS, Block DA, Lorig KR: Self-reports of health care utilization compared to provider records. J Clin Epidemio/ 2001, 54:136-141.

26. Haro JM, Salvador-Carulla L: The SOHO (Schizophrenia Outpatient Health Outcome) study: implications for the treatment of schizophrenia. CNS Drugs 2006, 20:293-301.

27. Luft B, Taylor D: A review of atypical antipsychotic drugs versus conventional medication in schizophrenia. Expert Opin Pharmacother 2006, 7:1739-1748

28. Nasrallah HA: Atypical antipsychotic-induced metabolic side effects: insights from receptor-binding profiles. Mol Psychiatry 2008, 13:27-35.

29. Stahl SM: Describing an atypical antipsychotic: receptor binding and its role in pathophysiology. Prim Care Companion J Clin Psychiatr 2003, 5(Suppl 3):9-13.

30. Lacro J, Dunn L, Dolder C, Leckband SG, Jeste DV: Prevalence of and risk factors for medication nonadherence in patients with schizophrenia: a comprehensive review of recent literature. J Clin Psychiatry 2002, 63:892-908.

\section{Pre-publication history}

The pre-publication history for this paper can be accessed here: http://www.biomedcentral.com/1471-244X/12/20/prepub

\section{doi:10.1186/1471-244X-12-20}

Cite this article as: DiBonaventura et al:: A patient perspective of the impact of medication side effects on adherence: results of a crosssectional nationwide survey of patients with schizophrenia. BMC Psychiatry 2012 12:20.

\section{Submit your next manuscript to BioMed Central and take full advantage of:}

- Convenient online submission

- Thorough peer review

- No space constraints or color figure charges

- Immediate publication on acceptance

- Inclusion in PubMed, CAS, Scopus and Google Scholar

- Research which is freely available for redistribution

Submit your manuscript at www.biomedcentral.com/submit
C Biomed Central 